\title{
Multiple-relaxation-time Lattice Boltzmann model for flow and convective heat transfer in lid driven cavity with porous obstacle
}

\author{
Abderrahmane Bourada ${ }^{1, *}$, Asma Ouahouah ${ }^{1}$, Kaoutar Bouarnouna $^{1}$, Karim Ragui $^{1}$, Abdelkader Boutra ${ }^{1,2}$, \\ and Youb Khaled Benkahla ${ }^{1}$ \\ ${ }^{1}$ Laboratory of Transfer Phenomena, FGMGP, USTHB, 16111 Bab Ezzouar, Algeria \\ ${ }^{2}$ Ecole Supérieure des Sciences Appliquées, 16001 Casbah, Algeria
}

\begin{abstract}
In this work, we study numerically a problem of mixed convection in lid driven square cavity, filled with air $(\operatorname{Pr}=0.71)$, whose upper wall is movable and kept at constant cold temperature $\mathrm{T}_{\mathrm{C}}$. The cavity contains a porous obstacle of height $h$ and width $b$, placed on the bottom wall maintained at a constant hot temperature $\mathrm{T}_{\mathrm{H}}$. The side walls are adiabatic. Darcy-Brinkmann-forchheimer model is used for modelling the momentum equations in porous medium. This numerical study is based on the multiple relaxation time lattice Boltzmann method (MRT -LBM). The D2Q9 two-dimensional model is adopted to the dynamic part, while the D2Q5 model is applied for the thermal part. The objective of the study is to analyze the effect of Darcy number $\left(10^{-1} \preccurlyeq \mathrm{Da} \preccurlyeq 10^{-5}\right)$, Richardson number $(0.01 \preccurlyeq \mathrm{Ri} \preccurlyeq 100)$ and the aspect ratio $\mathrm{w}=\mathrm{b} / \mathrm{H} \quad(0.2 \preccurlyeq \mathrm{w} \preccurlyeq 1)$ on the hydrodynamic and thermal characteristics in the cavity through the velocity and temperature as well as the average Nusselt number. The results obtained show a considerable effect of these parameters on the structure of the flow and on the heat exchange in the cavity.
\end{abstract}

\section{Introduction}

Mixed convection involving porous media is a phenomenon of considerable importance encountered in several natural and industrial processes. These applications include the extraction of geothermal energy, convection inside the Earth's mantle, electronic chip cooling, storage of spent fuel from nuclear power plants, drying... Etc. Given the complexity of these configurations, the numerical approach is very often used to solve the general equations governing the transfer of flux and heat.

In-depth studies have been carried out over the years, which characterize mixed convection involving porous media for different geometries. Chakravarty et al. [1] numerically studied mixed convection in an enclosure containing a heat-generating porous bed under the influence of background injection. Gibanov et al. [2] analyzed the effect of the inclined uniform magnetic field on mixed convection in a lid driven cavity having a saturated horizontal porous layer of a ferrofluid. Chattopadhyay et al. [3] studied mixed convection in a porous, double-walled movable cavity driven by sinusoidal heat. Astannina et al. [4] used a nanofluide to improve heat transfer in a movable-wall cavity with two porous layers in mixed convection. Liu and $\mathrm{He}$ [5] simulated double diffusion mixed convection in a fluid saturated porous medium by the multiple relaxation time lattice Boltzmann method. Taghizadeh and Asaditaheri [6] studied heat transfer and entropy generation on laminar mixed convection in a lid driven inclined cavity with a circular porous cylinder.

This paper deals with the numerical simulation of a mixed convection in a lid driven cavity, with porous obstruction and filled by a Newtonian fluid using the multiple relaxation time lattice Boltzmann method
(MRT-LBM). The D2Q9 two-dimensional discrete 9speed model was adopted to simulate the dynamic aspect of the problem, while the D2Q5 model is developed for the thermal field.

\section{Physical Problem}

Figure 1 provides a schematic overview of the twodimensional problem discussed in this study. A square cavity, containing air $(\mathrm{Pr}=0.71)$, which the upper wall is movable and maintained at a constant cold temperature $\mathrm{T}_{\mathrm{c}}$. This cavity contains a porous obstacle of height $\mathrm{h}=$ $0.5 \mathrm{H}$, width $\mathrm{b}$ and porosity equal to $\varepsilon=0.8$, mounted on the lower wall maintained at a constant hot temperature $\mathrm{T}_{\mathrm{h}}$. The side walls are adiabatic. The thermo-physical properties of the investigated fluid are assumed to be constant, except the density variation, in the buoyancy term, which follows the Boussinesq approximation [7]. We introduce the following dimensionless variables

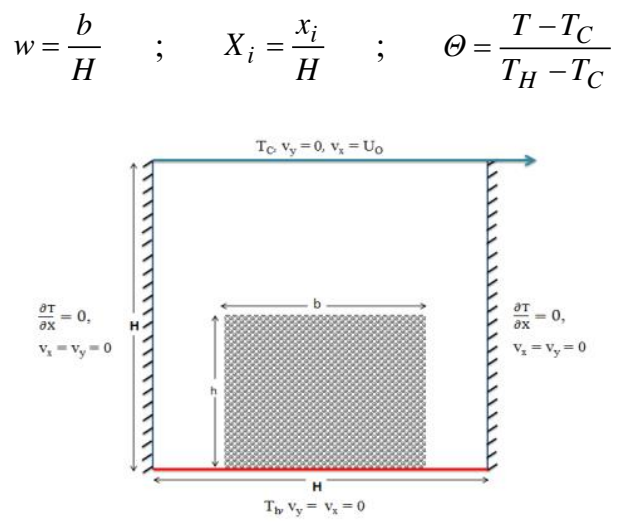

Fig. 1. Physical problem and boundary conditions.

\footnotetext{
* Corresponding author: abderrahmanebourada@gmail.com
} 


\section{Lattice Boltzmann method (LBM-MRT)}

Lattice Boltzmann approach uses the particle distribution functions $f(x, t)$, which signifies the probability of the presence of a large number of particles at site $x$ and time $\mathrm{t}$ in the mesoscopic system. Consequently, the geometry is covered by lattices which include a system of particles with symmetrical properties to satisfy the macroscopic domain with the rotation invariance. Generally, LBM includes two phases; the first phase is streaming in that a group of particles transfer on the lattice link according to the directional velocities by which the velocity space is described. The other step is collision where particles on the same lattice redistribute and relax into their quasiequilibrium. The overall lattice Boltzmann equation is defined as follows. These two steps are described by the following equation:

$$
f_{j}\left(x+v_{j} \Delta t, t+\Delta t\right)=f_{j}(x+t)+\Omega_{j} f_{j}(x+t), \quad i=0,1, \ldots, 8
$$

Where $\Omega_{\mathrm{j}}$ is the collision operator, representing the variation of the distribution function due to particle collisions.

In order to model the two-dimensional velocity field in the considered domain, the D2Q9 model on a square grid, of step $\Delta \mathrm{x}=\Delta \mathrm{y}=1$, was applied. The fluid particles move from one node of the gate to the neighboring node with discrete velocities, data ej, Peng [8]:

$$
\begin{aligned}
& e_{0}=(0,0) \\
& e_{1}=(1,0) ; e_{2}=(0,1) ; e_{3}=(-1,0) ; e_{4}=(0,-1) \\
& e_{5}=(1,1) ; e_{6}=(-1,1) ; e_{7}=(-1,1) ; e_{8}=(1,-1)
\end{aligned}
$$

The thermal field, meanwhile, has been described according to the model D2Q5 (network of five discrete temperatures).

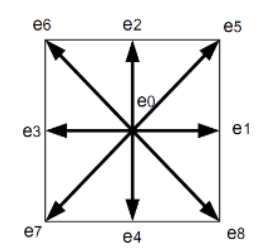

Fig. 2. D2Q9 Model.

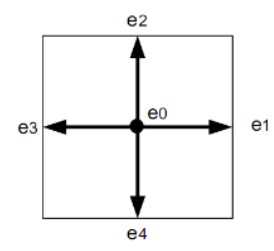

Fig. 3. D2Q5 Model.

The linearization of this operator around the local equilibrium distribution function brings a significant simplification of the LBM method.

On each domain node, the nine moments associated with the nine distribution functions are linked by the following linear transformation Mezrhab [9]:

$$
m=M . f
$$

Where the matrix $M$ is of order 9 .

During the colliding step which is local in space, three moments are preserved (the density and the momentum, in the longitudinal direction and the transversal direction), the six remaining moments, not conserved, are calculated from a simple equation of linear relaxation towards the equilibrium values which depend on the conserved quantities, Tekitek [10] :

$$
m_{k}^{*}(x, t)=\left(1-s_{k}\right) m_{k}(x, t)+s_{k} m_{k}^{e q}
$$

Where;

$\mathrm{s}_{\mathrm{k}}$ is the relaxation rate, $\mathrm{s}_{\mathrm{k}}=\Delta \mathrm{t} / \tau$

$\tau_{\mathrm{k}}$ is the relaxation time,

$m_{k}^{*}$ is the moment after collision and $m_{k}^{e q}$ is the equilibrium moment value.

For a reason of stability, relaxation rates verify double inequality $0 \leq \mathrm{s}_{\mathrm{k}} \leq 2$.

The kinematic viscosity of the fluid can be defined later as:

$$
v=\frac{1}{3}\left(\frac{1}{s_{8}}-\frac{1}{2}\right)
$$

The new distribution functions $f^{*}$ are calculated from the new moment's $\mathrm{m}^{*}$ :

$$
f^{*}=M^{-1} m^{*}
$$

On the Macroscopic scale, the mass density and the speed vector are given as follows:

$$
\begin{gathered}
\rho=\sum_{j=0}^{8} f_{j}(x, t) \\
u\left(x_{i}, t\right)=\frac{1}{\rho} \sum_{j=0}^{8} v_{j} f_{j}\left(x_{i}, t\right)
\end{gathered}
$$

The thermal transfer between the hot wall of the cavity and the cold fluid is characterized by the local $(\mathrm{Nu})$ and mean $\left(\mathrm{Nu}_{\text {avg }}\right)$ Nusselt numbers:

$$
N u=-\left.\frac{\partial \Theta}{\partial y}\right|_{y=0} \quad \text { and } \quad N u_{\text {avg }}=\int_{0}^{1} N u d x
$$

\subsection{Boundary Conditions}

For the model D2Q9 (velocity field), The condition " bounce back " is used to specify boundary conditions on solid walls:

$$
\begin{aligned}
& \text { upper wall: } f_{4}=f_{2}, f_{7}=f_{5}, f_{8}=f_{6} \\
& \text { lower wall: } f_{2}=f_{4}, f_{5}=f_{7}, f_{6}=f_{8} \\
& \text { left wall: } f_{1}=f_{3}, f_{5}=f_{7}, f_{8}=f_{6} \\
& \text { right wall: } f_{3}=f_{1}, f_{7}=f_{5}, f_{6}=f_{8}
\end{aligned}
$$

For model D2Q5 (thermal field): 
The condition of "Bounce Back" is imposed on the isolated walls:

left wall: $g_{1}=g_{3}$

right wall: $g_{3}=g_{1}$

and in the isothermal walls, we applied:

upper wall: $g_{4}=2$. delta.tc $-g_{2}$

lower wall: $g_{2}=2$.delta.tc $-g_{4}$

where; delta $=\alpha /\left[2\left(\mathrm{~s}_{1}-0.5\right)\right]$

\section{Code validation and grid independence}

The calculation code has been successfully validated with the numerical work of Liu et al. [11], for the case of a porous square cavity, differentially heated (cold right wall and hot left wall) with adiabatic vertical walls. This cavity of porosity equal to 0.4 is filled with a Newtonian fluid $(\operatorname{Pr}=1)$.

Table 1 show a good agreement between average Nusselt numbers obtained from this work and those of Liu et al. [11], for different numbers of Darcy and Rayleigh.

Table 1. Comparison of Average Nusselt number for various Da and Ra with $\varepsilon=0.4$ and $\operatorname{Pr}=1$.

\begin{tabular}{|c|c|c|c|c|c|c|}
\hline $\mathrm{Da}$ & $\mathrm{Ra}$ & {$[12]$} & {$[13]$} & $\begin{array}{c}\text { Liu et } \\
\mathrm{al}[11]\end{array}$ & $\begin{array}{c}\text { Present } \\
\text { code }\end{array}$ & $\begin{array}{c}\text { Ecart } \\
\%\end{array}$ \\
\hline \multirow{4}{*}{$10^{-2}$} & $10^{3}$ & 1.010 & 1.008 & 1.007 & 1.008 & 0.20 \\
\cline { 2 - 7 } & $10^{4}$ & 1.408 & 1.367 & 1.362 & 1.357 & 0.37 \\
\cline { 2 - 7 } & $10^{5}$ & 2.983 & 2.998 & 3.009 & 3.057 & 2.42 \\
\hline \multirow{3}{*}{$10^{-4}$} & $10^{5}$ & 1.067 & 1.066 & 1.067 & 1.066 & 0.10 \\
\cline { 2 - 7 } & $10^{6}$ & 2.550 & 2.603 & 2.630 & 2.597 & 0.23 \\
\cline { 2 - 7 } & $10^{7}$ & 7.810 & 7.788 & 7.808 & 7.792 & 0.57 \\
\hline \multirow{3}{*}{$10^{-6}$} & $10^{7}$ & 1.079 & 1.077 & 1.085 & 1.077 & 0.74 \\
\cline { 2 - 7 } & $10^{8}$ & 2.970 & 2.955 & 2.949 & 2.935 & 0.71 \\
\cline { 2 - 7 } & $10^{9}$ & 11.46 & 11.395 & 11.610 & 12.15 & 4.44 \\
\hline
\end{tabular}

Prior to the simulations, the mesh sensitivity analysis was performed to ensure a grid-independent solution. Table 2 shows the effect of the mesh on the average Nusselt number of the hot horizontal wall, at $\mathrm{Ri}=1$ ( with $\mathrm{Gr}=10^{4}$ ), $\mathrm{Da}=10^{-1}$, and $\mathrm{w}=0.6$. Based on the checks performed, the uniform mesh size of $121 \times 121$ nodes was selected for the rest of the simulations.

Table 2. Grid independence study at $\mathrm{Ri}=1, \mathrm{Da}=10^{-1}, \mathrm{w}=0.6$.

\begin{tabular}{|c|c|c|c|c|c|}
\hline Grid & 81 & 101 & 121 & 141 & 181 \\
\hline $\mathrm{Nu}_{\text {avg }}$ & 5.7007 & 5.7047 & 5.7066 & 5.7067 & 7.7067 \\
\hline$(\%)$ & - & 0.701 & 0.033 & 0.001 & 0 \\
\hline
\end{tabular}

\section{Results and discussion}

\subsection{Influence of Richardson number on streamlines and isotherms}

Figure 4 shows the streamlines and isotherms at $\mathrm{Da}=10^{-}$ 2 and $\mathrm{W}=0.6$, for different values of the Richardson number.

The streamlines indicate the presence of a main cell occupying the center of the cavity. The circulation inside this cell is governed by the direction of the translational movement of the upper wall. In this case, we have a clockwise circulation with a nucleus on the porous obstacle. The circulation inside the porous obstacle is low due to additional resistance of the porous matrix.

In the case of the dominant forced convection (Fig. 4 (a) and fig. 4 (b)); Two small cells appear at the lower corners of the cavity. The streamlines are tightened near the mobile wall (the upper wall), denoting the thinness of the thickness of the hydrodynamic boundary layer and the great parietal gradients of velocity.

In the mixed and dominant natural convection modes (Fig. 4 (c) and fig. 4 (d) respectively), the visualization of the streamlines reveals the dominance of natural convection effects and buoyancy forces within the enclosure.

In addition, the nucleus of the convective cell is shifted to the right part of the cavity, in the case of the mixed and forced convection dominant due to the movement of the upper wall.

In the case of a dominant forced convection (Fig. 4 (e) and fig. 4 (f)), the isotherms illustrate the formation of a hot thermal plume near the left vertical wall due to the clockwise circulation inside the cavity. The central part of the cavity is at an almost uniform cold temperature, this is caused by the high velocity of the cold horizontal wall in the low Richardson numbers.

For mixed convection (Fig. 4 (g)), isotherms begin to deform in the upper part of the cavity. Near the hot wall, the isothermal lines remain parallel to this wall and are confined in the form of a thin boundary layer, while in the upper left and central part of the cavity they are extended. This indicates the birth of convection near the isothermal walls and in the upper left and central part the conduction is dominant.

However, the isotherms of the natural convection (Fig. 4 (h)) show a slight deformation with a tightening of the isotherms in the immediate vicinity of the horizontal walls, indicating the beginning of the pairing of a temperature gradient in these regions.

It should be mentioned that the movement of the upper wall induces a shear flow of the adjacent fluid, while natural convection evolves from the thermal gradient. Therefore, it is possible to conclude that the shear stress and the vertical temperature gradient increase the flow of fluid and the heat transfer inside the cavity. 


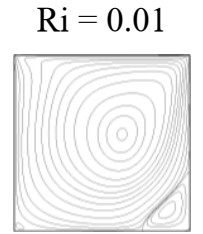

(a)

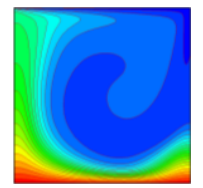

(e)

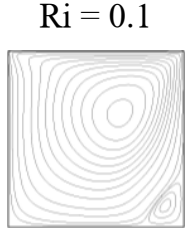

(b)

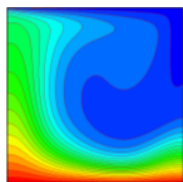

(f)

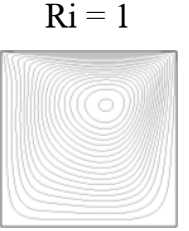

(c)

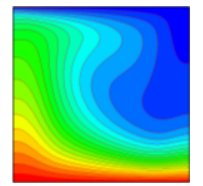

(g)

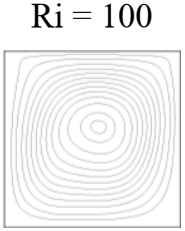

(d)

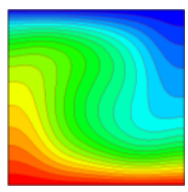

(h)
Fig. 4. Streamlines; (a), (b), (c) and (d), Isotherms;(e)-(f)-(g)(h); for different Richardson numbers. $\mathrm{Da}=0.01$ and $\mathrm{w}=0.6$.

\subsection{Influence of Darcy number on streamlines and isotherms}

The effect of Darcy number on the streamlines and isotherms is illustrated in Figure 5. This figure shows that Darcy has a significant effect on the flow and temperature characteristics in the cavity. For a high number of Darcy $\left(\mathrm{Da}=10^{-1}\right)$, where the porous medium is very permeable, the stream lines are characterized by a large central cell without disruption or significant braking. However, for lower Darcy numbers $\left(\mathrm{Da}=10^{-3}\right.$ et $\mathrm{Da}=10^{-4}$ ), the streamlines are distorted by passing through the porous obstacle which becomes less permeable. In a very low Darcy number $\left(\mathrm{Da}=10^{-5}\right)$, the porous obstacle acts as a solid and the main cell rotates over this obstacle causing the formation of two small cells adjacent to the lateral walls. A slight circulation of the fluid inside the porous medium is also remarkable at $\mathrm{Da}=10^{-5}$ with more observable disturbances.

Isotherms reflect the impact of Darcy's number on flux movement and show a lower heat transfer contribution by convention for lower Darcy values. We clearly note that for $\mathrm{Da}=10^{-1}$, Convection is more intense and the flow rates are greater. By reducing Darcy number, the convection heat transfer becomes lower.

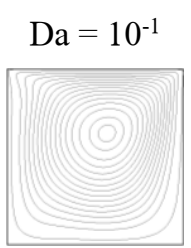

(a)

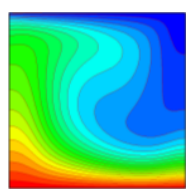

(e)

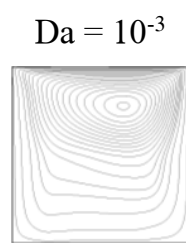

(b)

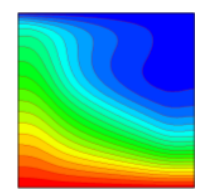

(f)

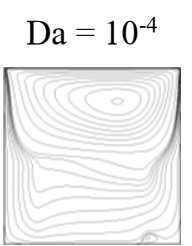

(c)

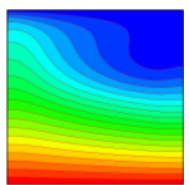

(g)

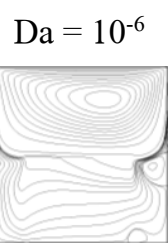

(d)

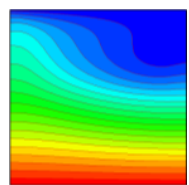

(h)
Fig. 5. Streamlines; (a), (b), (c) and (d) and Isotherms; (e)-(f)(g)-(h); for different values of Darcy number. $\mathrm{Ri}=1, \mathrm{w}=0.6$.

\subsection{Influence of the aspect ratio $W$, Darcy number and Richardson number on the heat transfer}

Figure 6 shows the evolution of heat transfer rate, calculated within the cavity at the hot horizontal wall, for different width of the porous obstacle, at different values of Darcy number, in mixed convection mode $(\mathrm{Ri}=1)$. For the highest Darcy numbers $\left(\mathrm{Da}=10^{-1}\right.$ and $\mathrm{Da}=10^{-}$ ${ }^{2}$ ), this rate, represented by the average Nusselt number, is optimal for a critical value of $w=0.4$. For higher values of the (reduced) width of the porous obstacle, the transfer rate decreases as the hot wall is in contact with less fluid. For the lowest Darcy numbers, the transfer rate increases with the decrease in the width of the obstacle, since the hot wall is in contact with fluid advantage.

Indeed, the best transfer is obtained with a number of Darcy equal to $10^{-1}$, since at this value the porous medium could be considered almost a fluid, thus promoting the thermal transfer between the cold fluid and the hot wall. This is not the case when $\mathrm{Da}=10^{-4}$ and $\mathrm{Da}=10^{-5}$, since in this case the obstacle behaves substantially as a non-permeable solid, inducing very low rates of heat transfer.

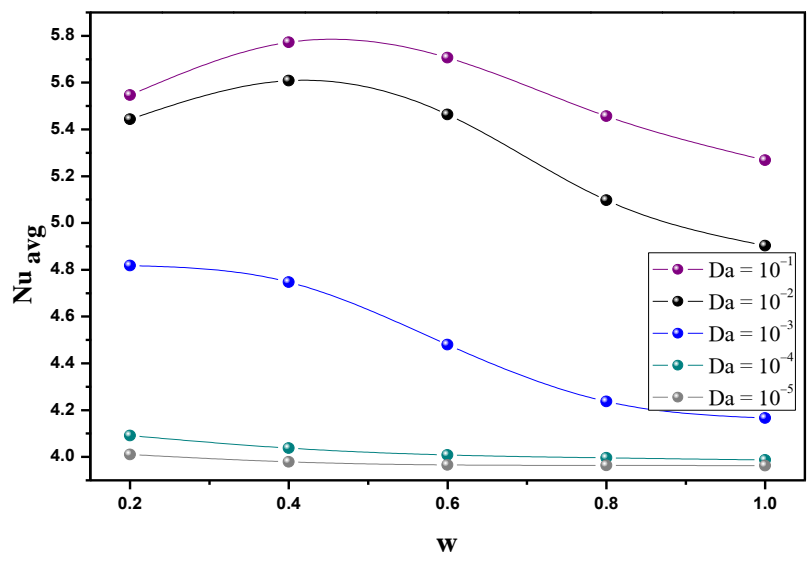

Fig. 6. Profiles of the mean Nusselt number at the heated wall for different values of $\mathrm{W}$ and Darcy number. $\mathrm{Ri}=1$.

The effect of Darcy number, for different Richardson numbers, on the evolution heat transfer to the hot wall is shown in Figure 7. It is noted that for a given Darcy number, the average Nusselt number decreases with the increase in Richardson number.

Indeed, simulations having been conducted for a fixed Grashof number $\left(\mathrm{Gr}=10^{4}\right)$, the increase in the Richardson number implies the reduction of the intensity of inertial forces responsible for the parietal shear and consequently to the increase in the rate of heat transfer.

The decrease in Darcy number and the increase in Richardson number lead to an increase in the thickness of the thermal boundary layer to the horizontal walls, resulting in the reduction of the average number of Nusselt.

In addition, the increase in Darcy's number improves transfer rates, especially in forced convection, where the greatest changes in the average Nusselt number are 
noted, compared with mixed and natural convection modes.

In conclusion, it can be said that the parietal shear, appearing in forced convection mode, promotes heat exchange.

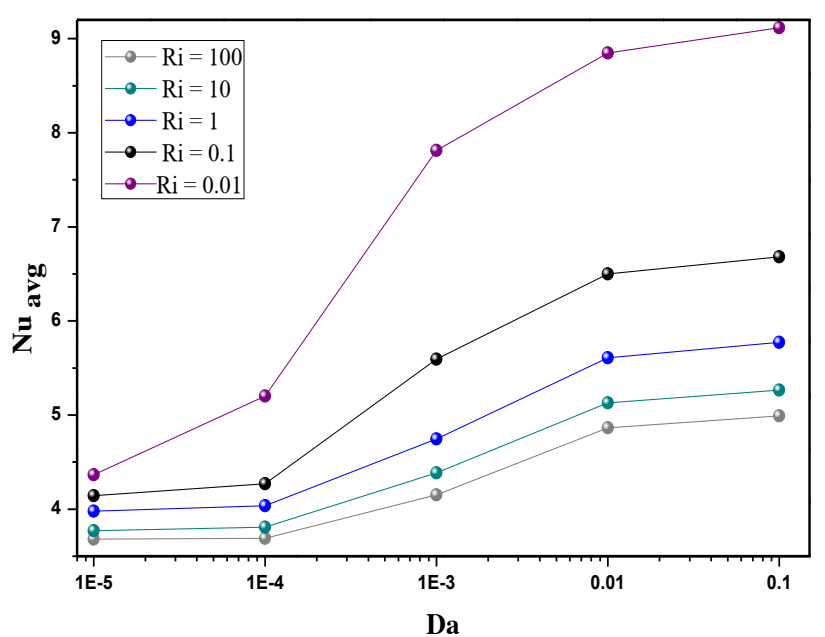

Fig. 7. Profiles of average Nusselt number at the heated wall for different Darcy and Richardson numbers. $\mathrm{w}=0.6$.

\section{Conclusions}

The study of mixed convection in a square, lid driven cavity with porous obstruction was performed by numerical simulation using a computational code based on the MRT-LBM. The effect of Darcy number, Richardson number, and the width of the porous obstacle on the flow of the fluid and the heat transfer was examined.

This study shows that the cooling of the hot wall surmounted by a porous obstacle is better when Darcy number is important.

Heat transfer is more important for the low Richardson numbers (in dominant forced convection mode).

For the important Darcy numbers the transfer rate is more important for the critical value of $\mathrm{w}=0.4$. While for the lower Darcy numbers, the transfer rate decreases with the increase of $\mathrm{w}$.

\section{References}

1. A. Chakravarty, P. Datta, K. Ghosh, S. Sen, A. Mukhopadhyay, , J. Heat Mass Transf. 117, 645, (2018)

2. N.S. Gibanov, M.A. Sheremet, H.F. Oztop, N. AbuHamdeh, Int. J. Heat Mass Transf, 114, 1086 (2017)

3. A. Chattopadhyay, S.K. Pandit, S.S. Sarma, I. Pop, Int. J. Heat Mass Transf., 93, 361, (2016)

4. M.S. Astanina, M.A. Sheremet, H.F. Oztop, N. AbuHamdeh, Int. J. Heat Mass Transf., 118, 527, (2018)

5. Q. Liu, Y. He, International Journal of Heat and Mass Transfer, 127, 497, (2018)

6. S. Taghizadeh, A. Asaditaheri, Int. J. Therm. Sci., 134, 242 (2018)
7. A. Bejan, Convection heat transfer, John Wiley and Sons, Inc., Hoboken, New jersey, USA, (2004)

8. Y. Peng, C. Shu, Y.T. Chew, Phys. Rev. E 68 026701. (2003)

9. A. Mezrhab, M. Bouzidi, P. Lallemand, Comp. Fluids, 33, 623, (2004)

10. M.M. Tekitek, PhD Thesis, Université de Paris sud, (2007)

11. Q. Liu, Y. He, Q. Li, W. Tao, Int. J. Heat Mass Transf., 73, 761, (2014)

12. P. Nithiarasu, K.N. Seetharamu, T. Sundararajan, Int. J. Heat Mass Transf., 40, 3955, (1997)

13. Z. Guo, T.S. Zhao, Numer. Heat Transf. B, 47, 157, (2005). 